\title{
Uniportal approach as an alternative to the three-portal approach to video-assisted thoracic surgery for primary spontaneous pneumothorax
}

\author{
Ryohei Yoshikawa, Natsumi Matsuura, Hitoshi Igai, Tomohiro Yazawa, Fumi Ohsawa, \\ Mitsuhiro Kamiyoshihara
}

Department of General Thoracic Surgery, Maebashi Red Cross Hospital, 389-1 Asakura-cho, Maebashi, Gunma 371-0811, Japan

Contributions: (I) Conception and design: R Yoshikawa; (II) Administrative support: H Igai; (III) Provision of study materials or patients: all authors;

(IV) Collection and assembly of data: all authors; (V) Data analysis and interpretation: R Yoshikawa; (VI) Manuscript writing: All authors; (VII) Final approval of manuscript: All authors.

Correspondence to: Ryohei Yoshikawa, MD. Department of General Thoracic Surgery, Maebashi Red Cross Hospital, 389-1 Asakura-cho, Maebashi, Gunma 371-0811, Japan. Email: ryoheyyoshikawa@gmail.com.

\begin{abstract}
Background: In recent years, uniportal video-assisted thoracic surgery (U-VATS) has been used for primary spontaneous pneumothorax (PSP). This study compared the perioperative outcomes of U-VATS and three-port VATS (3P-VATS) and sought to determine the risk factors for postoperative recurrence.

Methods: From October 2010 to February 2017, 232 patients with PSP undergoing surgical treatment were enrolled in this study. The patients were divided into two groups: U-VATS ( $\mathrm{n}=161)$ and 3P-VATS $(n=71)$ depending on the period of surgery. Retrospective analysis of the perioperative results and the risk factors for recurrence was performed.

Results: Both the operation time and duration of postoperative drainage were initially longer in the U-VATS group, but the difference gradually decreased such that ultimately there was no significant difference compared to the $3 \mathrm{P}-$ VATS group $(\mathrm{P}=0.10$ and $\mathrm{P}=0.12$, respectively). The duration of postoperative hospital stay and postoperative recurrence rate were not different between the two groups $(\mathrm{P}=0.084$ and $\mathrm{P}=0.44$, respectively). By multivariate analysis, the age (HR, 0.42, 95\% CI: 0.24-0.72, $\mathrm{P}<0.01)$ and number of bullae (single vs. multiple: HR, 0.03, 95\% CI: 0.002-0.54, P=0.02) were risk factors for recurrence.

Conclusions: The perioperative results and recurrence rate did not differ between the U-VATS and 3P-VATS groups, thereby demonstrating the non-inferiority of U-VATS. Postoperative risk factors for PSP recurrence were patient age and the number of bullae. Additional treatment may be needed to reduce recurrence in young patients with multiple bullae. Clinical registration number: The Institutional Review Board of Maebashi Red Cross Hospital (no. 2019-21).
\end{abstract}

Keywords: Uniportal video-assisted thoracic surgery; primary spontaneous pneumothorax (PSP); risk factors for recurrence

Submitted Sep 26, 2020. Accepted for publication Dec 10, 2020.

doi: $10.21037 /$ jtd-20-2928

View this article at: http://dx.doi.org/10.21037/jtd-20-2928

\section{Introduction}

Primary spontaneous pneumothorax (PSP) is a common disease in young males. Although chest tube drainage can resolve air leakage in most patients with PSP, approximately $50 \%$ of patients who receive this conservative treatment show recurrence of pneumothorax $(1,2)$. Therefore, surgical treatments, including bullectomy and blebectomy are recommended because they can decrease the likelihood of recurrence rate compared to conservative treatment (3). Although open thoracotomy had been used for the PSP 
since the 1990s, the use of video-assisted thoracic surgery (VATS) has increased over recent decades. Many reports describe how VATS is superior to open thoracotomy due to its low morbidity, lower invasiveness, low medical costs and better cosmetic outcomes (4). Several recent studies have demonstrated the feasibility of uniportal VATS (U-VATS) for PSP as a less-invasive treatment than the three-port VATS (3P-VATS) used in most previous investigations (3-5). However, the majority of studies comparing the surgical outcomes between U-VATS and 3P-VATS included only a small number of patients with PSP. Additionally, whether the surgical approach, specifically U-VATS or 3P-VATS, significantly affects the postoperative recurrence rate has not been determined. Based on the reduced invasiveness of a single skin incision, our group considered U-VATS as an alternative to 3P-VATS, assuming that neither the perioperative outcome nor the postoperative recurrence rate of U-VATS was inferior to $3 \mathrm{P}$-VATS. Therefore, in this study we retrospectively compared the perioperative results and postoperative recurrence rates of U-VATS bullectomy and 3P-VATS in patients with pneumothorax. We present the following article in accordance with the STROBE reporting checklist (available at http://dx.doi.org/10.21037/jtd-20-2928).

\section{Methods}

\section{Patients}

From October 2010 to February 2017, a total of 306 patients underwent surgery for PSP at our hospital. The surgical indications were as follows: (I) persistent air leakage, (II) recurrent episodes, (III) existence of bulla or blebs on computed tomography, (IV) severe collapse (from almost complete collapse to tension pneumothorax). The use of U-VATS for PSP began in May 2012. Therefore, patients with PSP between October 2010 and April 2012 underwent 3P-VATS. Patients over 40 years of age, those having a past history of ipsilateral pulmonary resection, and those requiring simultaneous bilateral surgery were excluded from this study. Finally, 232 patients with PSP were enrolled. In total, 161 patients were included in U-VATS group and 71 in the $3 \mathrm{P}-$ VATS group (Figure 1). To investigate the learning curve of U-VATS bullectomy, patients in the U-VATS group were further subdivided into two groups depending on whether surgery was performed during the early period (May 2012 to August 2014, n=81) or during the late period (September 2014 to May 2017, n=80).

\section{Preoperative treatment}

Pneumothorax was diagnosed based on chest X-ray or computed tomography findings. Patients with a moderately or severely collapsed lung underwent thoracic drainage on admission. Surgery was subsequently performed in those patients during the admission period if they met the abovedescribed criteria. Patients with a mildly collapsed lung underwent conservative management in the outpatient clinic and were scheduled for elective surgery. However, thoracic drainage was performed for those with a moderately or severely collapsed lung. A 16-Fr chest tube was typically used for thoracic drainage.

\section{Surgical technique}

Under general anesthesia using one-lung ventilation, the patients were placed in the lateral decubitus position. For 3P-VATS, a $15-\mathrm{mm}$ incision was made for stapling at the fifth intercostal space on the anterior axial line. A $7-\mathrm{mm}$ incision was subsequently made in the fifth or sixth intercostal space on the posterior axial line for a $5-\mathrm{mm}$ flexible thoracoscope (ENDOEYE FLEX LTF-S190-5: Olympus, Tokyo, Japan). Finally, a 5 -mm incision was made in the third intercostal space on the anterior axial line for grasping the bulla. For U-VATS, a 25-30-mm incision was made in the fifth or sixth intercostal space on the anterior axial line. The incision was extended for uniportal access in patients who underwent preoperative thoracic drainage. After placement of a $1-3-\mathrm{cm}$ wound retractor (Alexis Wound Retractor XXS ${ }^{\circledR}$; Applied Medical, Rancho Santa Margarita, CA, USA), we introduced a 5-mm flexible thoracoscope and instruments. We occasionally made a 3-mm incision for needlescopic forceps in the third intercostal space on the anterior axial line, if necessary. This incision did not require sutures at the end of the operation.

After detecting the target bulla, all regions of the lung were inspected, especially the posterior segment of lower lobe, to determine whether there were any additional bullae. Subsequently, the bulla was resected using endostaplers. After resection, polyglycolic acid felt (Neovei ${ }^{\circledR}$ Sheet; Gunze Co., Kyoto, Japan) was applied to the staple lines with $5 \mathrm{~mL}$ of fibrin glue sealant (Beriplast ${ }^{\circledR}$ P combi-set; CSL Behring GmbH, Marburg, Germany) to prevent postoperative air leakage and reduce the recurrence rate. Intraoperative pleurodesis (e.g., application of talc, pleurectomy, or pleural abrasion) was not performed. Finally, a $19 \mathrm{Fr}$ chest drain (Argyle ${ }^{\mathrm{TM}}$ Multi-Channel 


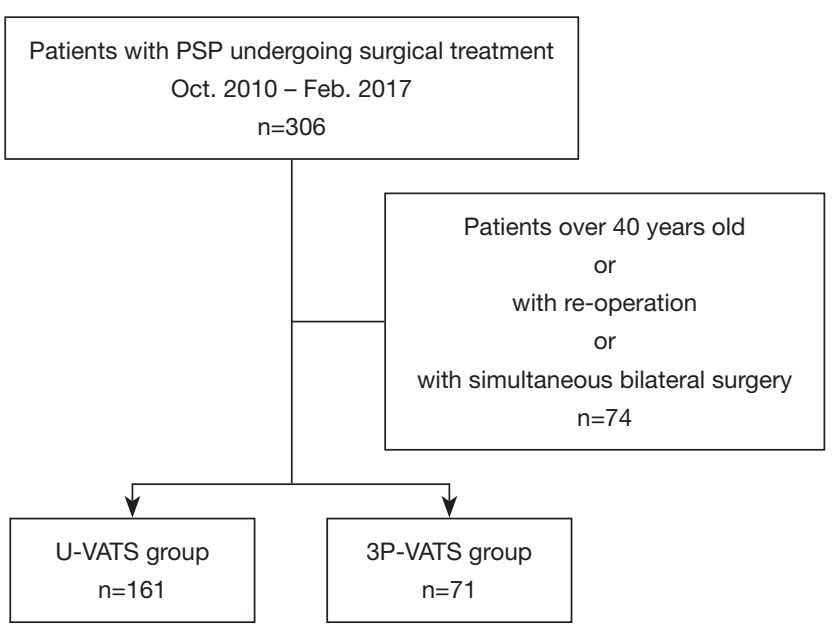

Figure 1 Summary of the 306 patients with pneumothorax. PSP, primary spontaneous pneumothorax; U-VATS, uniportal videoassisted thoracic surgery; 3P-VATS, three-port video-assisted thoracic surgery.

Drainage Catheter $\mathrm{S}^{\circledR}$; Covidien, Dublin, Ireland) was placed through the apex in an inverted $U$ shape from the anterior to posterior direction.

Young fellows or mid-career surgeons performed all of the operations in this series.

\section{Postoperative care and analgesia}

Analgesia was provided intravenously after surgery until the morning of postoperative day 1 , and orally thereafter. Drainage was performed with $-7 \mathrm{cmH}_{2} \mathrm{O}$ suction until the morning of postoperative day 1 . The chest tube was removed on postoperative day 1 when air leakage had ceased and chest X-ray demonstrated appropriate lung expansion. In case of postoperative air leak, $-7 \mathrm{cmH}_{2} \mathrm{O}$ suction was changed to a water seal without suction. On the day after chest tube removal, the patient was discharged if chest X-ray revealed ongoing and appropriate lung expansion.

All patients were followed for at least 2 years after surgery, either in the outpatient clinic or by telephone, including to determine whether pneumothorax relapse had been diagnosed in another hospital.

\section{Data collection and analysis}

Categorical variables are expressed as percentages and continuous variables as mean \pm standard division. Perioperative results were compared between the
3P-VATS and U-VATS groups by unpaired t-tests the $\chi^{2}$ test or Fisher's exact test. The recurrence rate was also compared between the two groups using the KaplanMeier methodology. To set a cut-off value for age, we used receiver operating characteristic (ROC) curve analysis. Univariate analysis was performed using the log-rank test to determine factors associated with recurrence. Cox proportional hazards regression was performed to identify risk factors for recurrence. Statistical significance was set at $\mathrm{P}<0.05$.

All statistical analyses were performed with EZR (Saitama Medical Center, Jichi Medical University, Saitama, Japan), which is a graphical user interface for R (R Foundation for Statistical Computing, Vienna, Austria) (6).

\section{Ethical statement}

The study was conducted in accordance with the Declaration of Helsinki (as revised in 2013). The study was approved by the Institutional Review Board of Maebashi Red Cross Hospital (no. 2019-21) and the requirement for informed consent for this retrospective analysis was waived.

\section{Results}

Table 1 shows the characteristics and perioperative results of the two groups. The proportion of males in the 3P-VATS group was significantly larger than in the U-VATS group $(\mathrm{P}=0.025)$ although there was no significant group difference in any other characteristic, including age $(\mathrm{P}=0.51)$ and affected side $(\mathrm{P}=0.78)$. Operation time and the duration of postoperative drainage were significantly longer in the U-VATS group than the 3 P-VATS group $(46.7 \pm 15.0$ and $38.1 \pm 17.5 \mathrm{~min}, \mathrm{P}<0.001,1.2 \pm 0.8$ and $1.0 \pm 0.1$ days, $\mathrm{P}=0.048$, respectively), and the proportion of patients with multiple bullae was significantly larger in the U-VATS group than in the $3 \mathrm{P}-\mathrm{VATS}$ group $(\mathrm{P}=0.027)$. There were no significant group differences in blood loss $(\mathrm{P}=0.51)$, length of postoperative hospitalization $(\mathrm{P}=0.084)$ or morbidity rate $(\mathrm{P}=0.093)$. Four patients in the U-VATS group required conversion to 3P-VATS because of adhesion $(n=3)$ or air leakage during the sealing test after resection of the bulla $(n=1)$. In this latter patient, it was difficult to repair the leakage site via the uniportal approach. Postoperatively, wound infection occurred in two cases in the 3P-VATS group, but was not seen in any case in the U-VATS group.

There were seven cases of postoperative recurrence. The differences in the recurrence rate between the U-VATS 
Table 1 Characteristics and perioperative outcomes of patients who underwent uniportal video-assisted thoracic surgery (U-VATS) and threeport video-assisted thoracic surgery (3P-VATS)

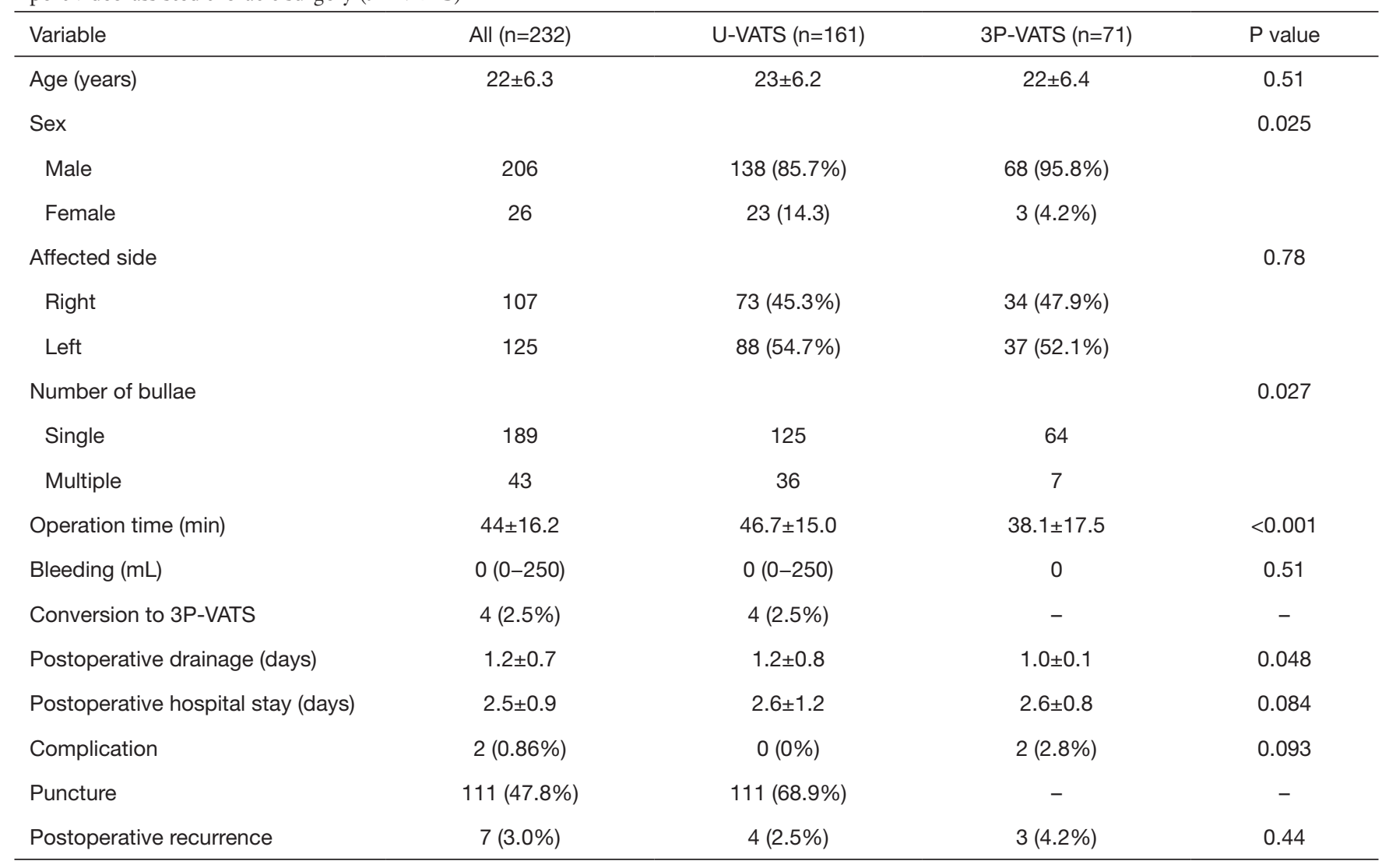

and 3P-VATS groups was not significant during the first 2 years postoperatively $(2.5 \%$ and $4.2 \%$, respectively, $\mathrm{P}=0.44$ ) (Figure 2). Three of the recurrences were re-treated by $3 \mathrm{P}-\mathrm{VATS}$ and all of them had new bullae around the staple line of last surgery. In one patient a drain was inserted followed by pleurodesis, while only drainage was performed in two patients. One patient was treated conservatively.

Figure 3 shows the ROC curve between age and recurrence rate. The cut-off value was set to 17 years old because the maximum area under the curve (AUC) value was seen at this age. Table 2 shows that age (HR: 0.42, 95\% CI: $0.24-0.72, \mathrm{P}<0.01)$ and the number of bullae (HR: 0.03, 95\% CI: $0.002-0.54, \mathrm{P}=0.02)$ were independent risk factors for recurrence.

In Table 3, the perioperative results of patients who underwent U-VATS during the early and late periods are compared. The operation time was significantly shorter during the late period than the early period $(42.2 \pm 13.4$ and $51.2 \pm 15.2 \mathrm{~min}$, respectively, $\mathrm{P}<0.01$ ), but there was no significant difference in any of the other perioperative outcomes. There was also no significant difference in the operation time or duration of postoperative drainage between the late U-VATS and 3P-VATS groups $(42.2 \pm 13.4$ and $38.1 \pm 17.5 \mathrm{~min}, \mathrm{P}=0.1$; and $1.1 \pm 0.7$ and $1.0 \pm 0.1 \mathrm{~min}$, $\mathrm{P}=0.12$, respectively).

\section{Discussion}

This study showed the non-inferiority of U-VATS compared to $3 \mathrm{P}-\mathrm{VATS}$ in terms of the perioperative outcome in patients treated for PSP. In addition, the surgical approach did not affect the likelihood of postoperative recurrence of pneumothorax.

Although 3P-VATS is now recognized as the standard surgical approach for PSP in several countries, especially Japan, the efficacy of U-VATS has recently been demonstrated. Masmoudi et al. (3) reported that U-VATS was a feasible, safe and reproducible treatment for PSP, based on analysis of large number of patients. In a metaanalysis comparing U-VATS and 3P-VATS for PSP, there 


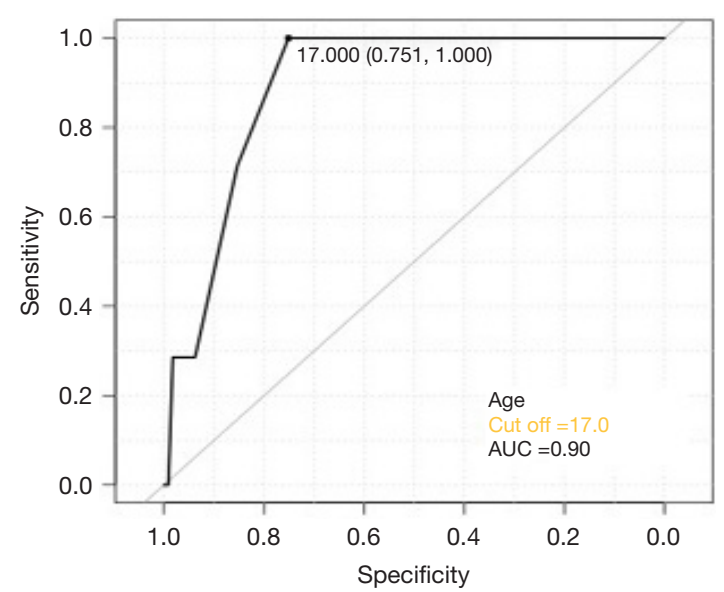

Figure 2 Recurrence rates in the U-VATS and 3P-VATS groups. U-VATS, uniportal video-assisted thoracic surgery; 3P-VATS, three-port video-assisted thoracic surgery.

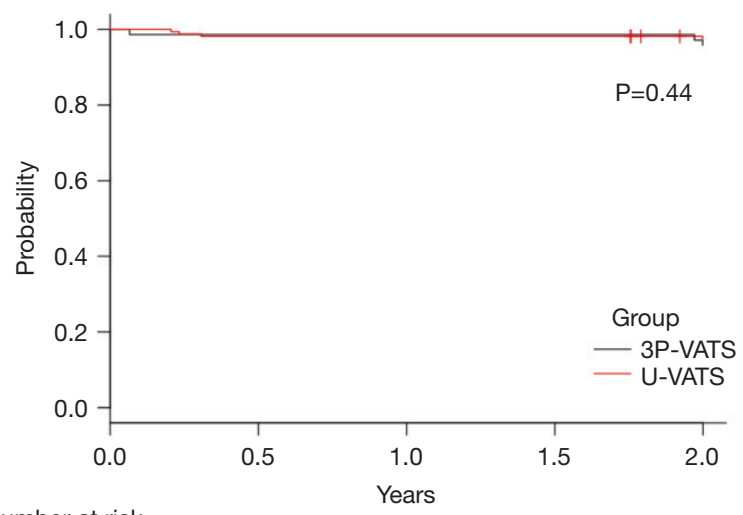

Number at risk 3P-VATS 71 U-VATS 161 70 158 $70 \quad 70$ 158 0
0

Figure 3 The receiver operating characteristic (ROC) curve of age and recurrence rate.

Table 2 Results of univariate and multivariate analyses of primary spontaneous pneumothorax recurrence.

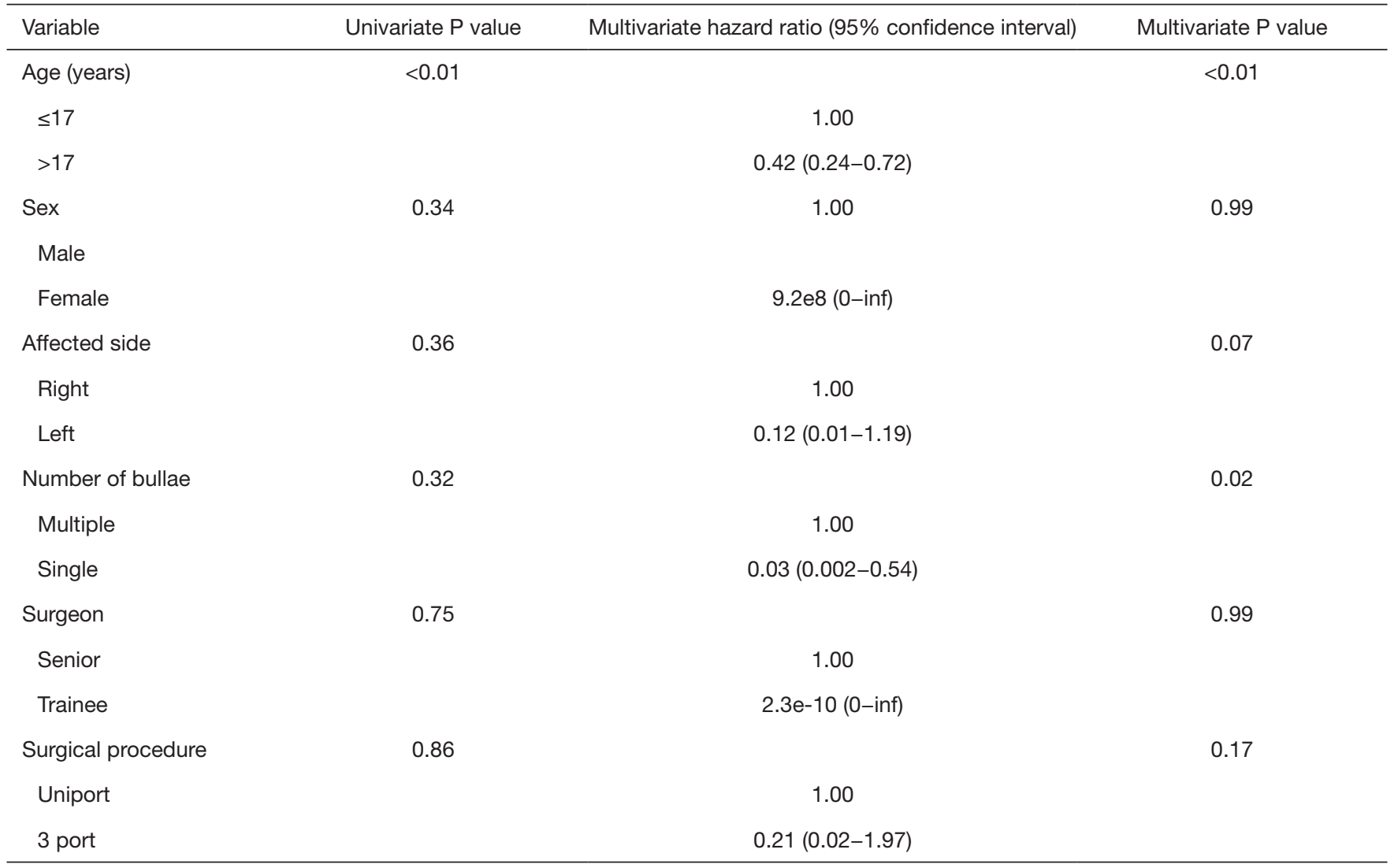

was no difference in operative time, mean duration of chest intubation, complications or recurrence rates $(7,8)$.

The operation time of the U-VATS group was significantly longer than that of the $3 \mathrm{P}-\mathrm{VATS}$ group in our series. In U-VATS, manipulating several surgical instruments via the same port and small incisions resulted 
Table 3 Perioperative outcomes of early (May 2012 to August 2014) and late (September 2014 to May 2017) U-VATS

\begin{tabular}{lccc}
\hline \multirow{2}{*}{ Variables } & \multicolumn{3}{c}{ U-VATS } \\
\cline { 2 - 3 } & Early period $(\mathrm{n}=81)$ & Late period $(\mathrm{n}=80)$ & P value \\
\hline Operation time $(\mathrm{min})$ & $51.2 \pm 15.2$ & $42.2 \pm 13.4$ & $<.001$ \\
Bleeding $(\mathrm{mL})$ & $0[0-250]$ & 0 & 0.32 \\
Postoperative drainage (days) & $1.3 \pm 1.0$ & $1.2 \pm 0.7$ & 0.33 \\
Postoperative hospital stay (days) & $2.5 \pm 1.0$ & $2.4 \pm 0.9$ & 0.48 \\
\hline
\end{tabular}

in interference between the thoracoscope and surgical instruments, which in turn resulted in technical difficulties and a prolonged operation time (9). Therefore, when necessary, a 3-mm incision into the third intercostal space on the anterior axial line was made for needlescopic forceps, to overcome such technical difficulties. A $3-\mathrm{mm}$ incision is not believed to increase the number of ports, like anchoring sutures (10), because such a small incision does not require suturing. This study also compared the perioperative results of patients undergoing U-VATS between early and lateperiod subgroups: the operation time of the latter group was significantly shorter. This demonstrated that improvement of operator skill with respect to U-VATS reduced the operation time. Our results showed that the operation time during the late U-VATS period was equivalent to that of 3P-VATS. Furthermore, the number of bullae was larger in the U-VATS group than in 3P-VATS group, which would have been a factor in the significantly longer operation time in the former group.

There have been several reports describing the efficacy of U-VATS for pneumothorax compared to 3P-VATS. Several authors reported no significant difference in recurrence rate between U-VATS and 3P-VATS groups $(11,12)$. However, the number of patients in these studies was small. A comparison of the perioperative results showed that the results of the U-VATS group were not inferior to those of the 3P-VATS group. Moreover, with a cohort of 232 patients, ours is the largest study to compare the two approaches. Therefore, our results constitute stronger evidence regarding the efficacy of U-VATS, including in terms of recurrence rate.

Although there have been reports on the postoperative recurrence rate of U-VATS (13), no analysis of risk factors for postoperative recurrence with U-VATS has been reported. In this study, age and the number of bullae were identified as risk factors. Patients with recurrence exhibited new bullae around the apex of the lung. Differences exist among patients in pleural vulnerability, which may also be involved in recurrence. West provided a hypothesis regarding the development of new bullae (14). Negative pressure increases gradually from the base to the apex of the lung, thus, in taller individuals, the alveoli are subject to strong distending pressure which seems to promote the development of new apical bullae. This pressure is expected to increase during periods of growth. In this study, ROC curve analysis concerning age and recurrence was performed (Figure 3); with a cut-off value of 17 years old, the AUC was at its maximum; it is interesting to note that the height of Japanese male plateaus at the age of 17 years (15). It seems that the younger the patient below the 17-year cut-off, the greater the increase in height after bullectomy, which is in turn related to the risk of recurrence.

Is there a way to prevent PSP recurrence? It is a wellknown that adhesion at the chest wall and reinforcement of the visceral pleura can prevent recurrence. Lee et al. (16) reported that pleural coverage was not inferior to mechanical pleurodesis and that patients with pleural coverage experienced significantly less pain. In this study, we applied polyglycolic acid sheets and fibrin glue around the staple line to reinforce the visceral pleura and promote adhesion. Our results indicate that it is important to widely cover the apex of the lung in young patients with multiple bullae. Mechanical pleurodesis was not performed in our patients but the procedure is probably common. While in our patients U-VATS was not inferior to 3P-VATS in terms of recurrence, the addition of mechanical pleurodesis may affect the outcome.

Due to the retrospective design of this study, neither postoperative pain nor patient satisfaction was assessed, as data were lacking. Qin et al. discussed the superiority of U-VATS in their meta-analysis. By contrast, in our patients the two approaches produced equivalent perioperative results and postoperative recurrence rates. However, since a single the $25-30 \mathrm{~mm}$ skin-incision is less invasive 
than multiple skin incisions, U-VATS is an appropriate alternative in the treatment of patients with PSP.

This study had some limitations. First, it was a retrospective, single-center study and due to the small number of recurrences the statistical power may have been insufficient. Second, we did not assess postoperative pain, paresthesia, or patient satisfaction. The meta-analysis that evaluated these variables suggested that U-VATS may have certain advantages over 3P-VATS $(7,8)$. Finally, the followup period was relatively short in this study, only 2 years. Thus, a prospective, randomized controlled trial with a longer follow-up period is required to validate the findings.

\section{Conclusions}

In conclusion, the perioperative results and recurrence rate did not differ between PSP patients treated by the U-VATS versus $3 \mathrm{P}-\mathrm{VATS}$, thereby demonstrating the non-inferiority of the former approach. Risk factors for PSP recurrence were older age and a higher number of bullae. Additional treatment (e.g., additional pleurodesis or covering the broad area around the staple line) may be needed to reduce the recurrence rate in young patients with multiple bullae.

\section{Acknowledgments}

Funding: None.

\section{Footnote}

Reporting Checklist: The authors have completed the STROBE checklist. Available at http://dx.doi.org/10.21037/ jtd-20-2928

Data Sharing Statement: Available at http://dx.doi. org/10.21037/jtd-20-2928

Peer Review File: Available at http://dx.doi.org/10.21037/jtd20-2928

Conflicts of Interest: All authors have completed the ICMJE uniform disclosure form (available at http://dx.doi. org/10.21037/jtd-20-2928). The authors have no conflicts of interest to declare.

Ethical Statement: The authors are accountable for all aspects of the work in ensuring that questions related to the accuracy or integrity of any part of the work are appropriately investigated and resolved. The study was conducted in accordance with the Declaration of Helsinki (as revised in 2013). The study was approved by the Institutional Review Board of Maebashi Red Cross Hospital (no. 2019-21) and the requirement for informed consent for this retrospective analysis was waived.

Open Access Statement: This is an Open Access article distributed in accordance with the Creative Commons Attribution-NonCommercial-NoDerivs 4.0 International License (CC BY-NC-ND 4.0), which permits the noncommercial replication and distribution of the article with the strict proviso that no changes or edits are made and the original work is properly cited (including links to both the formal publication through the relevant DOI and the license). See: https://creativecommons.org/licenses/by-nc-nd/4.0/.

\section{References}

1. Sahn SA, Heffner JE. Spontaneous pneumothorax. N Engl J Med 2000;342:868-74.

2. Sawada S, Watanabe Y, Moriyama S. Video-assisted thoracoscopic surgery for primary spontaneous pneumothorax: evaluation of indications and long-term outcome compared with conservative treatment and open thoracotomy. Chest 2005;127:2226-30.

3. Masmoudi H, Etienne H, Sylvestre. Three hundred fiftyone patients with pneumothorax undergoing uniportal (Single Port) video-assisted thoracic surgery. Ann Thorac Surg 2017;104:254-60.

4. Nachira D, Ismail M, Meacci E. Uniportal vs. triportal video-assisted thoracic surgery in the treatment of primary pneumothorax-a propensity matched bicentric study. J Thorac Dis 2018;10:S3712-9.

5. Bertolaccini L, Pardolesi A, Brandolini J. Uniportal videoassisted thoracic surgery for pneumothorax and blebs/ bullae. J Vis Surg 2017;3:107.

6. Kanda Y. Investigation of the freely available easy-touse software 'EZR' for medical statistics. Bone Marrow Transplant 2013;48:452-8.

7. Yang Y, Dong J, Huang Y. Single-incision versus conventional three-port video-assisted surgery in the treatment of pneumothorax: a systematic review and metaanalysis. Interact Cardiovasc Thorac Surg 2016;23:722-8.

8. Qin SL, Huang JB, Yang YL. Uniportal versus three-port video-assisted thoracoscopic surgery for spontaneous pneumothorax: a meta-analysis. J Thorac Dis 2015;7:2274-87. 
9. Igai H, Kamiyoshihara M, Ibe T. Single-incision thoracoscopic surgery for spontaneous pneumothorax using multi-degrees of freedom forceps. Ann Thorac Cardiovasc Surg 2014;20:974-79.

10. Son BS, Kim DH, Lee SK. Small single-incision thoracoscopic surgery using an anchoring suture in patients with primary spontaneous pneumothorax: a safe and feasible procedure. Ann Thorac Surg 2015;100:1224-9.

11. Chen CH, Lee Sy, Chang Ho. The adequacy of singleincisional thoracoscopic surgery as a first-line endoscopic approach for the management of recurrent primary spontaneous pneumothorax: a retrospective study. J Cardiothorac Surg 2012;7:99.

12. Tsuboshima K, Wakahara T, Matoba Y. Singleincision thoracoscopic surgery using a chest wall pulley for lung excision in patients with primary spontaneous

Cite this article as: Yoshikawa R, Matsuura N, Igai H, Yazawa T, Ohsawa F, Kamiyoshihara M. Uniportal approach as an alternative to the three-portal approach to video-assisted thoracic surgery for primary spontaneous pneumothorax. J Thorac Dis 2021;13(2):927-934. doi: 10.21037/jtd-20-2928 pneumothorax. Surg Today 2015;45:595-9.

13. Imperatori A, Rotolo N, Spagnoletti M. Risk factor for postoperative recurrence of spontaneous pneumothorax treated by video-assisted thoracoscopic surgery. Interact Cardiovasc Thorac Surg 2015;20:647-51.

14. West JB. Distribution of mechanical stress in the lung, a possible factor in localization of pulmonary disease. Lancet 1971;1:839-41.

15. Isojima T, Kato N, Ito Y. Growth standard chart for Japanese children with mean and standard deviation (SD) values based on the year 2000 national survey. Clin Pediatr Endocrinol 2016;25:71-6.

16. Lee S, Kim HR, Cho S. Staple line coverage after bullectomy for primary spontaneous pneumothorax: a randomized trial. Ann Thorac Surg 2014;98:2005-11. 